DOI: http://doi.org/10.21698/simi.2017.0013

\title{
STUDY ON NANOPARTICLES FLOW REDUCTION TESTS TO HWI PLANTS USING NUMERICAL SIMULATIONS
}

\author{
Mihai Lungu ${ }^{1, \text { a) }}$, Adrian Neculae ${ }^{1}$, Antoanetta Lungu ${ }^{1}$, Nicolae Strambeanu ${ }^{2}$, Daniel \\ $\operatorname{Arghiriade}^{2}$, Laurentiu Demetrovici ${ }^{2, \text { b) }}$ \\ ${ }^{1}$ Faculty of Physics, West University of Timisoara, 4 V. Parvan Blvd, Timisoara, Romania \\ ${ }^{2}$ Pro Air Clean Ecologic SA, 5 Sulina 5, Timisoara, Romania, ${ }^{a)}$ Imihai@ physics.uvt.ro, \\ ${ }^{b)}$ tehnic@proairclean.ro
}

\begin{abstract}
This study deals with the experimental evaluation of some relevant ash samples collected from the flue gas filters of a hazardous waste incinerator plant. The experimental analysis focuses on size, distribution and dielectric properties of the samples. The reported results reveal the presence of nanoparticles in the samples and the possibility to use dielectrophoresis for their handling. The study exploits also numerical simulations in order to investigate the behavior of nanoparticles with size range from 50 to $150 \mathrm{~nm}$, which is fructified as a micro channel - working unit used as a particulate trap. Based on the experimental data and a proposed mathematical model, the concentration profile of nanoparticles suspension inside a micro fluidic separation device is calculated and the performance of the device is analyzed in terms of three new specific quantities of separation process, called Recovery, Purity and Separation Efficiency.
\end{abstract}

Keywords: air pollution, dielectrophoresis, filtration rate, flue gas filtration, hazardous waste, incineration, microfluidic device, nanoparticle separation, $P M_{2.5}$, $P M_{1}$

\section{Introduction}

Timisoara $\left(45^{\circ} 46^{\prime} \mathrm{N}, 21^{\circ} 26^{\prime} \mathrm{E}\right)$ is a city located on the south-eastern edge of the Pannonia plain, and lies at an altitude of $85 \mathrm{~m}$, being one of the largest Romanian cities, with a population of over 300 thousands inhabitants and more than 170 thousands automobiles. The air quality in urban areas is determined by the intensity of emissions (Sbrizzai et al. 2005; Rickerby \& Morrison 2007). The principal sources of pollution are from transportation (emission from traffic), industrial and households. The main pollutants tend to be airborne particles, sulphur dioxide, nitrogen dioxide, carbon monoxide, ozone and volatile organic compounds (VOCs).

Health effects of air pollutants, i.e. nanoparticles, is an old issue in cities and urban areas around the world, but became evident during severe air pollution episodes in the first part of the 21- th century. Once the association between the existence of fine particles suspended in atmosphere and their negative impact on the human health has been proved clearly, the problem of the control of the toxic emissions has become of great scientific interest. Recent health effects studies have shown an association between existing levels of fine particles (size, concentration) and health effects such as increased respiratory illness, cardiopulmonary morbidity, and premature mortality (Minutolo et al. 2010; Rickerby \& Morrison 2007). 


\section{INTERNATIONAL SYMPOSIUM "THE ENVIRONMENT AND THE INDUSTRY", SIMI 2017, PROCEEDINGS BOOK}

From a public health standpoint, the size of a particle is as important as its composition, recent research showing that although raw materials may not be dangerous, they can become toxic under the form of nanoparticles. Although the nanoparticles have smaller masses than microparticles, their number is at least four orders of magnitude higher than the number of all other particles found in the flue gas. Incinerator-related activities result in the emission of a host of air pollutants that adversely affect public health and the environment, including nitrogen oxides $\left(\mathrm{NO}_{\mathrm{x}}\right)$, hydrocarbons (HC), particulate (PM), carbon monoxide (CO), which causes lung irritation and aggravates diseases such as asthma, chronic bronchitis, and emphysema (Chang \& Huang 2001). In this context, the problem of obtaining information about the composition and dispersion of different resulted combustion residues is a first and fundamental step. The transport process of the components is investigated numerically with the CloseView software. The input program data are the concentration and the chemical properties of the components detected experimentally in the combustion chimney. Our study takes into account the effects that the height of the combustion chimney, the velocity and the direction of the wind has on the dispersion process. The concentration profiles are calculated for a geometry specific to the city map of Timisoara. This analysis provides relevant information on the distribution of the pollutants and the most exposed zones of the city. In this paper we take a close view of pollutant dispersion in the environment, how the wind and atmospheric condition affects the dispersion for point like emission sources. As a result of highlighting for this micro pollutants by the mentioned method and considering that emissions sources are generally equipped with filters able to retain only micro particles, usual methods (retention and separation) have not been successful for the separation of nanoparticles (Lungu et al. 2010). The most promising technique for nanoparticle trapping and controlled spatial separation is a method based on dielectrophoresis (DEP), phenomenon in which a spatially non-uniform AC electric field induces a dipole moment in a dielectric particle. Hence, the particle undergoes a DEP motion under the resulting translational force. This force does not require electrically charged particles; the strength of the force depends on the medium and particle's electrical properties, particle's shape and size, and on the applied electric field amplitude and frequency (Pethig 2010; Neculae et al. 2012; Green et al. 2002).

\section{Materials and Methods}

Hazardous waste incineration is one of the widely used waste disposal technologies which both reduces waste volume and NOx amount and recovers energy as a last alternative of judicious use. This technology complies with specific regulations that establish a threshold for direct VOC emissions or other forms of spreading volatile, powder and other waste gases type at the stack of incineration plants.

Filtration of submicron particles suspended in flue gas is an important technological challenge, as in urban environment the burning processes including incinerators of waste or diesel emissions are responsible for the emission of a significant amount of nanoparticles (Sbrizzai et al. 2005). The presence in the environment of nanoparticle with size ranging from $50 \mathrm{~nm}$ to $200 \mathrm{~nm}$ has a profound impact on human health (Rickerby \& Morrison 2007). Once inhaled, due to their tendency to remain trapped in the inner respiratory ways, infiltrate into the blood and cannot be eliminated, because the macrophage cells cannot identify them. Inhaled nanoparticles may 


\section{INTERNATIONAL SYMPOSIUM "THE ENVIRONMENT AND THE INDUSTRY", SIMI 2017, PROCEEDINGS BOOK}

generate free radicals, affect the DNA and alter the genes, being responsible for mutagens and carcinogenic effects or causing a variety of lung-disease typologies. From a public health standpoint, the size of a particle is as important as its composition, recent research showing that although raw materials may not be dangerous, they can become toxic under the form of nanoparticles (Rickerby \& Morrison 2007; Minutolo et al. 2010). Although the nanoparticles have smaller masses than microparticles, their number is at least four orders of magnitude higher than the number of all other particles found in the flue gas. As a direct and immediate consequence, the filtration of nanoparticles is an important technological challenge, as they are produced in large quantities from material synthesis and combustion emission. Sources of polluting emissions are generally equipped with various filters that capture the micron particles, but permit nanoparticles to escape in atmosphere (Chang \& Huang 2001; Lungu et al. 2010). In many scientific and technical areas, a considerable interest is shown to the separation of nanoparticles in accordance with their physical or chemical characteristics. The methods utilizing electric fields are emerging as most promising techniques for nanoparticle manipulation, namely those based on dielectrophoresis (DEP): a phenomenon in which, under spatially nonuniform AC or DC electric fields, dielectric particles move because of the interaction of the dipole induced in the particle and the applied field gradient. DEP methods can be used in many forms to manipulate and more generally, control the position, orientation and velocity of submicron scale particles. DEP force does not require electrically charged particles; the strength of the force depends on the medium, particle's electrical properties, particle's shape and size, and on the applied electric field frequency (Pethig 2010; Neculae et al. 2012). Since the relative dielectric polarization of the nanoparticles depends on the driving frequency of the applied electric field, an alternating (AC) electric field is usually applied to generate DEP forces of different magnitudes and directions. In classical dielectrophoresis, the positive DEP force attracts particles into the regions of strong electric fields, while negative DEP force repels them from those regions (Green et al. 2002; Çetin \& Li 2011). More recently, using advanced microelectrode fabrication techniques, the technology has moved into the submicrometer world so that nanoparticles can now be characterized and separated. The manipulation of nanoparticles in microsystems by using DEP forces has many existing and potential applications, presenting the advantages of voltage-based control and dominance over other forces: in the range above a few millimeters, the electrical forces are rather ineffective, but in the micrometer (and submicrometer) scale the electrical forces dominate. Microfluidics involves the manipulation of nanoparticles within a microscale chip. Within microfluidic systems, the manipulation of nanoparticles is of fundamental importance, including patterning, focusing, sorting, trapping, handling and separation (Zhang et al. 2010).

For the modern hazardous waste incineration plants, the current standards provide strict limits both for $\mathrm{PM}_{2.5}$ and indirect nanoparticle emissions released as heavy metals that are more or less volatile at high temperatures (Strambeanu \& Demetrovici 2009; CD 438/2006).

The identification of the primary composition of stack emissions has led to the conclusion that some improved particulate retention systems were effective in removing about $99.99 \%$ of particles, with similar efficiency in reducing particles of smaller diameter (Buonanno et al. 2011). However, it has been found that the boiling 


\section{INTERNATIONAL SYMPOSIUM "THE ENVIRONMENT AND THE INDUSTRY", SIMI 2017, PROCEEDINGS BOOK}

points of incinerated elements have special significance: higher boiling point elements like were frequently identified in larger particles, while lower boiling point elements like arsenic and cadmium were more common in ultrafine particles whose toxicity level they increased (AEA 2012).

Table 1. Emission limits recommended for incinerators of hazardous waste and sludge in treatment stations (CD 438/2006)

\begin{tabular}{|c|c|c|c|c|c|}
\hline & Code & \multicolumn{4}{|l|}{ Name } \\
\hline NFR Source Category & $\begin{array}{l}\text { 5.C.1.b.i, } \\
\text { 5.C.1.b.il, } \\
\text { 5.C.1.b.iv }\end{array}$ & \multicolumn{4}{|c|}{ Industrial waste incineration including hazardous waste and sewage sludge } \\
\hline Fuel & \multicolumn{5}{|l|}{ NA } \\
\hline Not applicable & \multicolumn{5}{|l|}{$\mathrm{HCH}$} \\
\hline Not estimated & \multicolumn{5}{|c|}{$\begin{array}{l}\mathrm{NH}_{3}, \mathrm{Cr}, \mathrm{Cu}, \mathrm{Zn}, \mathrm{Se}, \text { Benzo(a)pyrene, Benzo(b)fluoranthene, Benzo(k)fluoranthene, } \\
\text { Indeno(1,2,3-cd)pyrene, PCBs }\end{array}$} \\
\hline Pollutant & Value & Unit & Lower & Upper & Reference \\
\hline $\mathrm{NO}_{\mathrm{x}}$ & 0.87 & $\mathrm{~kg} / \mathrm{Mg}$ waste & 0.087 & 8.7 & European Commission (2006) \\
\hline $\mathrm{CO}$ & 0.07 & $\mathrm{~kg} / \mathrm{Mg}$ waste & 0.007 & 0.7 & European Commission (2006) \\
\hline NMVOC & 7.4 & $\mathrm{~kg} / \mathrm{Mg}$ waste & 0.74 & 74 & Passant (1993) \\
\hline $\mathrm{PM}_{2.5}$ & 0.004 & $\mathrm{~kg} / \mathrm{Mg}$ waste & 0.0004 & 0.1 & US EPA (1996) applied on TSP \\
\hline $\mathrm{BC}$ & 3.5 & $\%$ of $\mathrm{PM}_{2.5}$ & 1.8 & 7 & Olmez et al. (1988) \\
\hline $\mathrm{Pb}$ & 1.3 & g/Mg waste & 0.48 & 1.9 & Theloke et al. (2008) \\
\hline Cd & 0.1 & $\mathrm{~g} / \mathrm{Mg}$ waste & 0.048 & 0.15 & Theloke et al. (2008) \\
\hline $\mathrm{Hg}$ & 0.056 & g/Mg waste & 0.04 & 0.08 & European Commission (2006) \\
\hline As & 0.016 & $\mathrm{~g} / \mathrm{Mg}$ waste & 0.01 & 0.019 & Theloke et al. (2008) \\
\hline $\mathrm{Ni}$ & 0.14 & g/Mg waste & 0.048 & 0.19 & Theloke et al. (2008) \\
\hline $\mathrm{PCDD} / \mathrm{F}$ & 350 & $\mu \mathrm{g}$ I-TEQ/Mg waste & 0.5 & 35000 & UNEP (2005) \\
\hline Total 4 PAHs & 0.02 & $\mathrm{~g} / \mathrm{Mg}$ waste & 0.007 & 0.06 & Wild (1995) \\
\hline $\mathrm{HCB}$ & 0.002 & g/Mg waste & 0.0002 & 0.02 & Berdowski et al. (1997) \\
\hline
\end{tabular}

While incineration of any kind of waste has a small contribution to anthropogenic nanoparticle emissions (CD 438/2006; AEA 2012; Buonanno et al. 2011), there are studies that attempt to establish a direct correspondence between thermal waste elimination and the statistical parameters of population health (Buonanno et al. 2011; BS 2005).

For instance, particle size distribution by specific particle weight may cause serious errors, especially in hazardous waste incinerators equipped with catalytic systems for reducing nitrogen oxides, which may actually double the $\mathrm{PM}_{2.5}$ emissions (BS 2005). Most stack emissions contain ultrafine particles of the most dangerous type and fabric filters with Teflon membrane are ineffective against such particles, especially those under 200-300 nm which are considered extremely harmful to health.

Most current modelling methods do not take into consideration the particles that are reformed from atmospheric gas emissions far from the incinerator stack, although a number of studies have shown how in $95 \%$ of cases ultrafine heavy metal particles combine with polynuclear aromatic hydrocarbons (PAH) to produce $\mathrm{PM}_{3}$ or smaller conglomerates. PAHs are toxic, mutagenic, teratogenic and cancer-causing. Such combinations increase the lung cancer risk by almost eight times (BS 2005).

Timisoara $\left(45^{\circ} 46^{\prime} \mathrm{N}, 21^{\circ} 26^{\prime} \mathrm{E}\right)$ is a city on the South-Western border of the Pannonian Plain. Situated at an altitude of $85 \mathrm{~m}$, it is one of Romania's largest cities, with over 300,000 inhabitants and more than 170,000 cars. Starting with 2001, a hazardous 


\section{INTERNATIONAL SYMPOSIUM "THE ENVIRONMENT AND THE INDUSTRY", SIMI 2017, PROCEEDINGS BOOK}

waste incineration plant built in compliance with the EC construction and operation standards has been in operation in the city's southern industrial area.

The study investigates the behavior of nanoparticles with size ranging from 50 to 150 $\mathrm{nm}$ in a DEP-based microsystem, which consists in a microchannel-working unit of a particulate trap. After a brief presentation of the theoretical model, we proceed to the experimental determination of the key characteristics of the particles suspended in flue gas: the size distribution of the submicron particles collected from the flue gas filters of a hazardous waste incinerator plant and their dielectric properties are evaluated. Based on the experimental data and the proposed model, the concentration profile of nanoparticle suspension inside the microfluidic separation device is calculated and the performance of the device is analyzed in terms of new specific quantity of separation process, called Filtration rate. The final goal of the study is to identify a suitable way to predict accurately the nanoparticle entrapment and find the optimal values of the control parameters for separation process, to be useful in designing of microfluidic devices for separating nanoparticles from combustion gases.

\section{Theoretical consideration}

The theoretical approach of particulate diffusion in the atmosphere is based on Fick's second law:

$$
\frac{d c}{d t}=D \frac{d^{2} c}{d r^{2}}+G(c)
$$

This equation is the mathematical expression of concentration $c$ in time $t$ in a spacetime coordinate point $(r, t)$. The $G(c)$ function defines the nanoparticle content in the sedimentable particle flow released at the stack. The diffusion coefficient $(D)$ is proportional with the temperature $(T)$ and indirectly proportional with the amount of diffusing particles, as well as their form. The diffusion coefficient has a large range in gases: $D \sim 10^{-5} \mathrm{~m}^{2} / \mathrm{s}$. For colloidal spherical particles, it is calculated with the Stokes-Einstein relation:

$$
D=\frac{k T}{6 \pi}(p 1+p 2) \square
$$

where: $k$-Boltzmann's constant; $h$-dynamic viscosity coefficient; $p_{1,2}$ - average radii of sedimentable / non-sedimentable particles.

The integration of equation (1) can lead to precise analytical solutions, but it requires certain initial conditions that can frequently differ from those required for finding solutions for similar differential equations without physical-chemical signification.

In such cases, the numerical integration methods usually lead to experimentally reproducible models with satisfactory results. The transport of settled particles experimentally defined below was numerically shaped through the original CloseView program (Strambeanu et al. 2016). The time averaged DEP force acting on a spherical particle situated in an AC electric field can be written as (Morgan \& Green 2003):

$$
\left\langle\mathbf{F}_{D E P}\right\rangle=2 \pi a^{3} \varepsilon_{m} K_{R}(\omega) \nabla\left(\left|\nabla V_{R}\right|^{2}+\left|\nabla V_{I}\right|^{2}\right)
$$




\section{INTERNATIONAL SYMPOSIUM "THE ENVIRONMENT AND THE INDUSTRY", SIMI 2017, PROCEEDINGS BOOK}

where we denoted by $a$ the particle radius, $\omega$ the angular field frequency, and $V_{R}$, respectively $V_{I}$ the real and imaginary part of the electric potential phasor, $\tilde{V}=\ddot{V_{R}}+J V_{I}$, with $j=\sqrt{-1}$. For a homogeneous medium, the electric potential satisfy the Laplace equation: $\Delta^{2} \tilde{V}=0 . K_{R}(\omega)$ is the real part of the complex quantity

$$
\tilde{K}(\omega)=\left(\tilde{\varepsilon}_{p}-\tilde{\varepsilon}_{m}\right) /\left(\tilde{\varepsilon}_{p}+2 \tilde{\varepsilon}_{m}\right),
$$

named as Clausius-Mossotti (CM) factor, which is a measure of the effective polarizability of the particle. $\tilde{\varepsilon}_{p}$ and $\tilde{\varepsilon}_{m}$ are the complex dielectric permittivities of particle and medium. The complex permittivity is defined as $\tilde{\varepsilon}=\varepsilon-j(\sigma / \omega)$, where $\sigma$ is the electrical conductivity.

The $\mathrm{CM}$ factor depends on the dielectric properties of the particle and medium and on the frequency of the applied field; at low frequencies the sign is determined by the electrical conductivities of the particle and the medium and at higher frequencies by the permittivities. The variation in this factor results in a frequency-dependent dielectrophoretic force that is unique for a particular type of particle. Therefore, DEP is an effective tool for separating particles, based solely on their dielectric properties and size. When the sign of $K_{R}$ is positive, the particle is attracted to the locations of electric field intensity maxima and repelled from the minima, phenomenon known as positive dielectrophoresis (pDEP). The opposite occurs when $K_{R}$ is negative, situation referred to as negative dielectrophoresis (nDEP).

A typical DEP-based separation device has parallel interdigitated bar electrodes placed on the bottom surface is illustrated in Fig. 1.

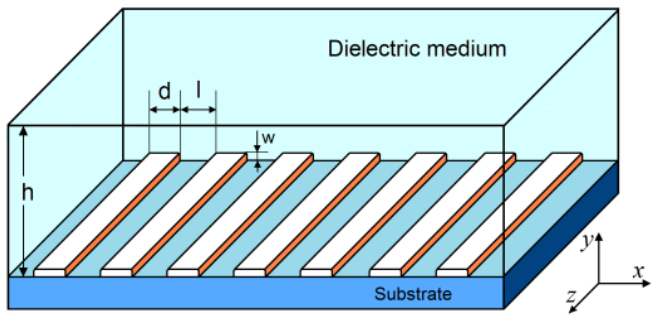

Figure 1. Schematic representation of experimental device used for DEP separation

In most of the reported models, due to the symmetry of the geometry and considering the electrodes much longer than their width, the problem is treated in two dimensions and the electrodes' height is neglected. In our study, in order to have a more realistic description of the experimental device, a 3D geometry and a detailed description of the electrodes shape are used. Anyway, taking into account the periodic distribution of the electrodes, the numerical calculations of the DEP force and the concentration field can be performed considering as computational domain only a so called "basic unit cell", which fully describes the entire system, except the vicinity of the walls. The geometry of the computational domain, together with the associated boundary 


\section{INTERNATIONAL SYMPOSIUM "THE ENVIRONMENT AND THE INDUSTRY", SIMI 2017, PROCEEDINGS BOOK}

conditions necessary to solve the Laplace equation for electric potentials $V_{R}$ and $V_{I}$ are presented in Fig. 2.

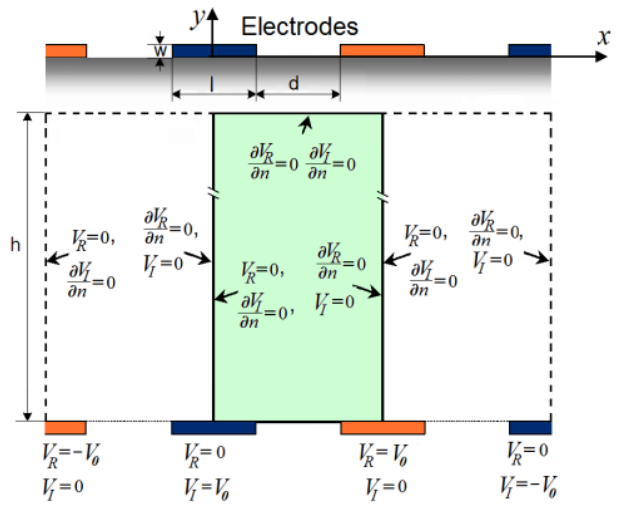

Figure 2. The geometry of the computational domain and the associated boundary conditions for the electric potentials. The solid lines indicate the basic unit cell

The macroscopic behavior of a suspension of spherical particles of radius $a$ in a fluid of viscosity $\eta$ is modeled by considering the mechanical equilibrium between an external force $\mathbf{F}$ (DEP force in this case) and the Stokes drag force. For small particles (i.e. nanoparticles), the dynamics of the system is governed by the following system of equations (Neculae et al. 2012):

$$
\begin{gathered}
\mathbf{v}=\mathbf{u}+\frac{2 a^{2}}{9 \eta} \mathbf{F}, \\
\frac{\partial C}{\partial t}+\nabla \cdot \mathbf{j}=0, \text { where } \mathbf{j}=C \mathbf{v}-D \nabla C
\end{gathered}
$$

Here $\mathbf{u}$ and $\mathbf{v}$ are the fluid and particle velocities, $t$ is the time, $\mathbf{j}$ is the particle flux, $D$ is the diffusion coefficient of the particles, and $C$ is the particle volumic concentration. The fluid flow field inside the separation device, $\mathbf{u}$, is calculated by solving the classical Navier-Stokes equation in the compressible case, together with the corresponding boundary conditions. For the obtained DEP-force and fluid flow field, the particle concentration is evaluated by numerically integrating equations (5a) and (5b). This calculated particle concentration field gives information at a local scale, showing how the particles are attracted on the margins of electrodes and the influence of the main parameters of the problem on this process. For the analysis of the filtration process we define the novel quantity named Filtration rate $(F)$, which describes the process in terms of nanoparticles entrapment at the electrodes, related to the concentration distribution:

$$
F=\frac{C_{\text {input }}-C_{\text {output }}}{C_{\text {input }}}=1-C_{\text {output }} / C_{\text {input }} \quad[\%]
$$




\section{INTERNATIONAL SYMPOSIUM "THE ENVIRONMENT AND THE INDUSTRY", SIMI 2017, PROCEEDINGS BOOK}

where $C_{\text {input }}$ and $C_{\text {output }}$ are the mean concentrations of suspended nanoparticles at the input and the output surfaces of the device, respectively, as schematically sketched in Fig. 3.

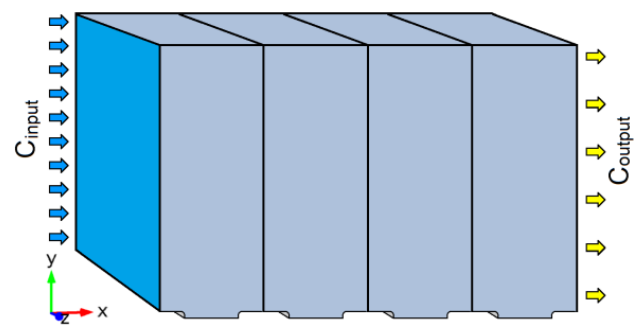

Figure 3. Schematic representation of the separation device revealing the parameters used for defining the Filtration rate

This proposed quantity gives the global information on the filtration process, and can be used in order to evaluate the efficiency of the filtration process.

At the end of this theoretical section is important to conclude that the electrokinetic forces depend in a complex manner on system dimensions, frequency, field, etc. In separation systems, the buoyancy force can be sometimes significant but often the magnitude of this force is much lower than the other forces acting on nanoparticles.

\section{Results and Discussion}

\section{Emissions measurements}

Incineration activities generate atmospheric pollutant emissions with a harmful effect on health and the environment, including NOx, hydrocarbons (HC), particulates, carbon monoxide (CO) that cause lung damage and aggravate diseases like asthma, chronic bronchitis, emphysema etc. (Zhiqiang et al. 2000; Popescu et al. 2011). Consequently, obtaining information about the composition and dispersion of various combustion components is the first essential step to take against polluting emissions. The system of continuous monitoring of the particulates at stack is shown in Table 2. The system measures the emissions every two minutes. The analyser sends the data to the central command-control unit that compares it to the admissible values and adjusts the flow chart.

The particle transport was modelled numerically with the original CloseView software. The study quantified the effects that the height of the stack identified as point source and the wind speed and direction have on the continuous particle dispersion process. The concentrations were calculated for the geometry specific to the city map of Timisoara, for the last five years (2011-2016). The graphic representation of the particulate dispersion statistics for the last five years reveals the areas of the city that are mostly exposed to nanoparticle emissions at the incinerator stack (Figure 4). These are represented by the SE-NW sectors, reproducible with 3$5^{\circ}$ deviations every year. 


\section{INTERNATIONAL SYMPOSIUM "THE ENVIRONMENT AND THE INDUSTRY", SIMI 2017, PROCEEDINGS BOOK}

Table 2. Data about the measuring and control equipment

\begin{tabular}{c|cc}
\hline Parameter & Method & Equipment \\
\hline Particulate & Triboelectric & DURAG D-RX250 System \\
Flow rate & Prandtl congestion tube & DURAG D-RX250 System \\
Humidity & Capacimetry & Calibrated condenser \\
Temperature & Thermo-electrical & Thermocouples, thermal resistors \\
\hline
\end{tabular}

Some references indicate that the waste incineration plants generate relevant amounts of fine and ultrafine particulates, as the current standard value for particulate emissions $\left(10 \mathrm{mg} / \mathrm{Nm}^{3}\right)$ provides a filter retention rate of only $5-30 \%$ for $\mathrm{PM}_{2.5}$ and $0 \%$ for $\mathrm{PM}_{1}$ (BS 2005).

As a result, a fraction ranging within the above-mentioned limit values consists of non-sedimentable nanoparticles that can affect a city area far larger than that revealed by the statistical calculations of long-term particulate emissions which determine the particulate number and size, but not the particulate flow rate at the stack or the imissions (AEA 2012; BS 2005).

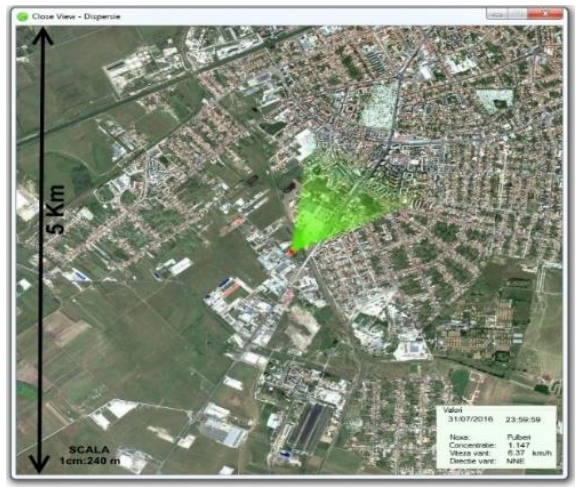

Figure 4. Prevailing particulate dispersion at the stack of the incineration plant (20112015)

Such considerations have underlined the necessity to investigate the technical possibilities of limiting nanoparticle emissions. These are to be applied at the emission point source represented by the flue gas evacuation system of the hazardous waste incinerator, in relation to the specific context of the area where the incinerator is located.

\section{Flue gas filtration}

In order to obtain relevant input data for the simulations, we investigated three probes, named as A, B and C, consisting in samples of ash resulted from the combustion of different wastes, collected monthly from filters of Pro Air Clean Timisoara hazardous waste incinerator, within a period of three months. These probes were analyzed from dimensional and dielectric point of view. For dimensional characterization, we prepared for each of the three probes a mixture of $5 \mathrm{mg}$ ash in $100 \mathrm{ml}$ distilled water at room temperature, and put it to rest for 20 minutes, in order to decant the 


\section{INTERNATIONAL SYMPOSIUM "THE ENVIRONMENT AND THE INDUSTRY", SIMI 2017, PROCEEDINGS BOOK}

microparticles. Then we collected the remained slurry liquid and analyzed the particle size/concentration distribution using a Nano Sight LM 10 nanoparticle visualization system, based on nanoparticle-tracking analysis method. Fig. 4a-4c illustrate the particle size/concentration distribution for the three probes. The distribution diagram indicates that it contains four significant groups of nanoparticles, having sizes of 55 $\mathrm{nm}, 100 \mathrm{~nm}, 155 \mathrm{~nm}$, and $275 \mathrm{~nm}$, respectively.

These diagrams show that the gas resulting from the combustion of waste contains nanoparticles, nanoparticles having radii ranging from 50 to $200 \mathrm{~nm}$, considered potentially harmful for human health. For each of the three probes one observe higher concentrations for particles with radii of about $50 \mathrm{~nm}, 100 \mathrm{~nm}$, and $150 \mathrm{~nm}$, values that will be considered relevant in our future analysis. The efficiency of the filtration process can be evaluated by calculating the Filtration rate (6) for different values of problem's parameters. The computation is performed using an iterative procedure: the output concentration in one unit cell is considered the input concentration for the next unit cell, in order to describe the cumulative effect of the filtration inside the dielectrophoretic device. This type of analysis allows an estimation of the necessary number of cells (or electrodes) in order to obtain a certain desired filtration rate, when the other parameters of the problem are fixed. The results presented in Fig. 5-a show that in the case of particle having size of $100 \mathrm{~nm}$, a desired filtration rate of $90 \%$ can be obtain by using about 30 electrodes when applying a voltage of $24 \mathrm{~V}$, about 60 electrodes for $18 \mathrm{~V}$, and nearly 200 electrodes for an applied voltage of $12 \mathrm{~V}$.

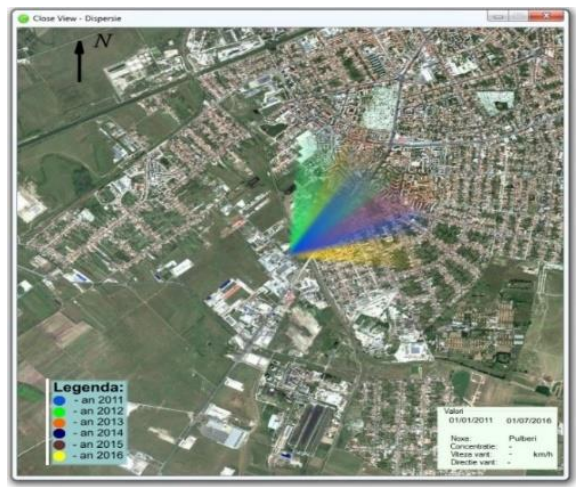

Figure 5. Change in average annual angular dispersion depending on atmospheric conditions (2011-2015)

When we analyze the effect of particle radiion the filtration capacity, the results presented in Fig. 8-b predict that, for example, when the applied voltage is $18 \mathrm{~V}$, particles of $150 \mathrm{~nm}$ are completely captured after 10 electrodes, for particles of 100 nm we need about 150 electrodes for the complete capture, while for the particles of $50 \mathrm{~nm}$ are captured less than $60 \%$ even if one use devices with 250 electrodes.

The next step of our research activity will focus on the validation of the theoretical model. Some preliminary but promising experimental results were already obtained. 


\section{INTERNATIONAL SYMPOSIUM "THE ENVIRONMENT AND THE INDUSTRY", SIMI 2017, PROCEEDINGS BOOK}

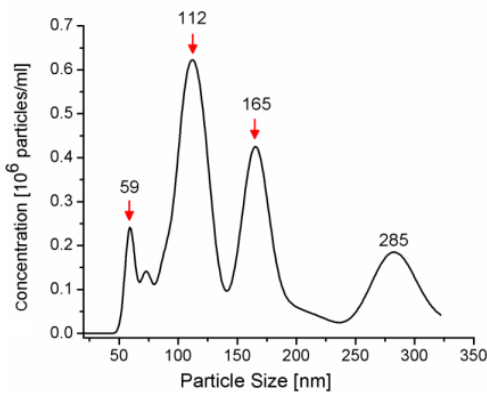

(a)

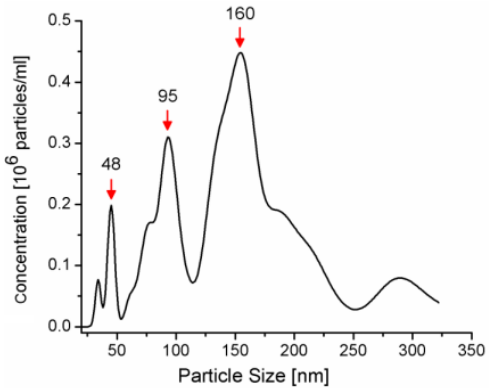

(b)

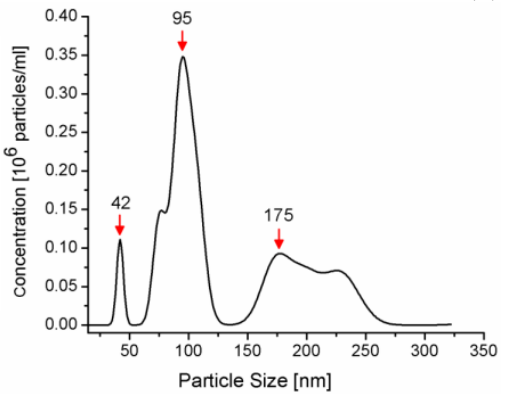

(c)

Figure 6. Particle size/concentration distribution diagram for probes a, b and c, after 20 minutes of decantation

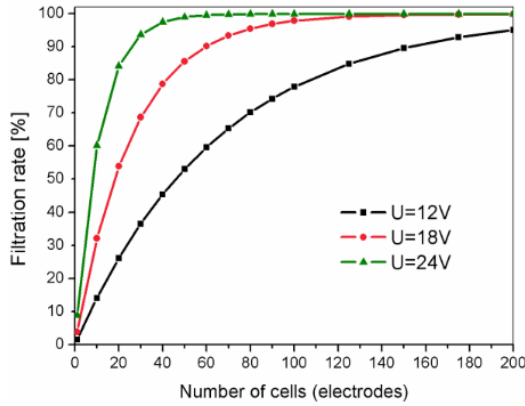

(a)

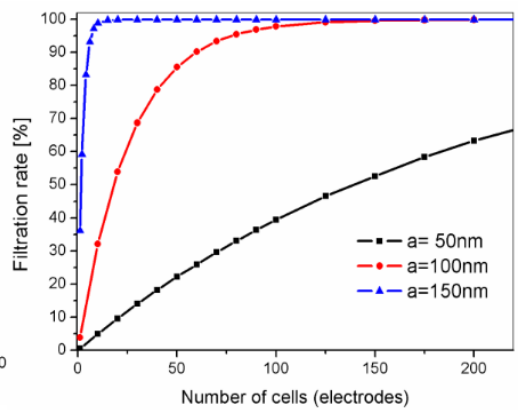

(b)

Figure 7. Calculated filtration rate versus number of cells for a) particles with $a=100$ $\mathrm{nm}$ at three different applied voltages and b) particles with three different radii at an fixed applied voltage of $V_{0}=18 \mathrm{~V}(d=l=100 \mu \mathrm{m}, w=2 \mu \mathrm{m})$

The tests performed with a DEP-based separation device having $l=d=100 \mu \mathrm{m}$ and $\mathrm{H}=1000 \mu \mathrm{m}$ reveal that in the absence of the applied voltage the particles are not at all attracted to the electrodes (Figure 6-a), while once applied an AC voltage of $12 \mathrm{~V}$ the dielectrophoretic effect appears (Figure 6-b). More than that, the concentration of 


\section{INTERNATIONAL SYMPOSIUM "THE ENVIRONMENT AND THE INDUSTRY", SIMI 2017, PROCEEDINGS BOOK}

captured particles clearly diminishes while we depart from the input region, which is in concordance with our simulations.

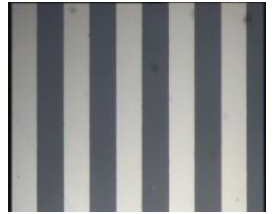

(a)

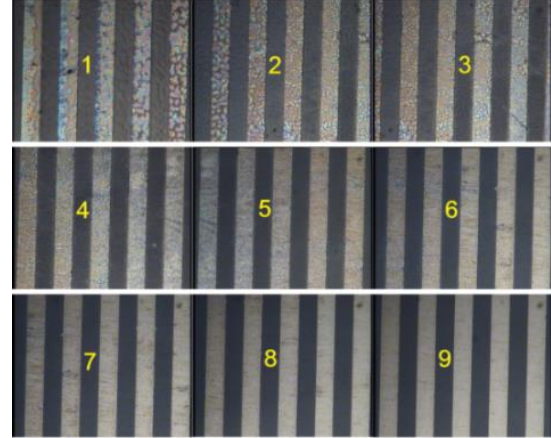

(b)

Figure 8. DEP-based separation device with $l=d=100 \mu \mathrm{m}$; a) before fumigation, b) after fumigation, successive snapshots, $U=12 \mathrm{~V}, \mathrm{AC}, 50 \mathrm{~Hz}$, time of fumigation $\mathrm{t}=30 \mathrm{~s}$

After this qualitative validation, a quantitative evaluation of the concentration of nanoparticles captured at the electrodes, at different distances from the input of the device, is necessary in order to give a solid validation of the model.

\section{Conclusions}

We introduced a theoretical model and applied it to a physically realistic problem, and then we predict a novel mechanism of particle entrapment, which is not only fundamentally different from the one conventionally known but also, is proven to be efficient at small scales. The particle concentration distributions for a typical interdigitated electrodes array are calculated using the proposed theoretical model. The efficiency of the manipulation process is discussed in terms of Filtration rate, for different values of particle radius and applied voltage. The optimal parameters of the separation process can be determined through a detailed numerical study performed in the frame of the proposed mathematical model and used for designing more efficient separation devices.

\section{Acknowledgments}

This work was supported by a grant of the Romanian National Authority for Scientific Research, CNCS - UEFISCDI, project number PN-II-ID-PCE-2011-3-0762.

\section{References}

AEA 2012, AEA ED57607001 Report, Review of Research into Health Effectsof Energy from Waste Facilities, January 3, 2012

BS 2005, 'The Health Effects Waste Incineration, 4th Report of the British Society for Ecological Medicine, 8-10, December 2005'. vol. no. pp.

Buonanno, G, Stabile, L, Avino, P \& Belluso, E 2011, 'Chemical, dimensional and morphological ultrafine particle characterization from a waste-to-energy plant'.

Waste Management, vol. 31, no. 11, pp. 2253-2262.

CD 438/2006, Comisional Decision CE 438, February 28, 2006. 


\section{INTERNATIONAL SYMPOSIUM "THE ENVIRONMENT AND THE INDUSTRY", SIMI 2017, PROCEEDINGS BOOK}

Çetin, B \& Li, D 2011, 'Dielectrophoresis in microfluidics technology'. Electrophoresis, vol. 32, no. 18, pp. 2410-2427.

Chang, MB \& Huang, CK 2001, 'Characteristics of energy flow in municipal solid waste incinerator'. Journal of environmental engineering, vol. 127, no. 1, pp. 78-81.

Green, NG, Ramos, A \& Morgan, H 2002, 'Numerical solution of the dielectrophoretic and travelling wave forces for interdigitated electrode arrays using the finite element method'. Journal of Electrostatics, vol. 56, no. 2, pp. 235-254.

Lungu, M, Neculae, A \& Bunoiu, M 2010, 'Some considerations on the dielectrophoretic manipulation of nanoparticles in fluid media'. Journal of optoelectronics and advanced materials, vol. 12, no. 12, pp. 2423-2426.

Minutolo, P, Sgro, L, Costagliola, M, Prati, M, Sirignano, M \& D’Anna, A 2010, 'Ultrafine particle emission from combustion devices burning natural gas'. Chem Eng Trans, vol. 22, no. pp. 239-244.

Morgan, H \& Green, NG 2003, AC electrokinetics, Research Studies Press,

Neculae, A, Biris, CG, Bunoiu, M \& Lungu, M 2012, 'Numerical analysis of nanoparticle behavior in a microfluidic channel under dielectrophoresis'. Journal of Nanoparticle Research, vol. 14, no. 10, pp. 1154.

Pethig, R 2010, 'Dielectrophoresis: Status of the theory, technology, and applications'. Biomicrofluidics, vol. 4, no. 2, pp. 022811.

Popescu, F, Ionel, I, Belegante, L, Lontis, N \& Cebrucean, V 2011, 'Direct measurements an numerical simulations issues in airport air quality'. International Journal of Energy and Environment, vol. 5, no. 3, pp. 410-417.

Rickerby, D \& Morrison, M 2007, 'Nanotechnology and the environment: A European perspective'. Science and Technology of Advanced Materials, vol. 8, no. 1, pp. 19-24.

Sbrizzai, F, Faraldi, P \& Soldati, A 2005, 'Appraisal of three-dimensional numerical simulation for sub-micron particle deposition in a micro-porous ceramic filter'. Chemical Engineering Science, vol. 60, no. 23, pp. 6551-6563.

Strambeanu, N, Gherebeanu, D, Arghiriade, D, Bumbu, B, Mihailescu, M \& Popa, AD 2016, 'Statistical study of the atmospheric dispersion of sulphur and nitrogen oxides at the stack of a special waste incineration plant located in the southern part of Timisoara', International Symposium "The Environment and The Industry", National Research and Development Institute for Industrial Ecology, Bucharest, pp. 206-213.

Strâmbeanu, N \& Demetrovici, L Year, 'Published Comparative calculation of incineration costs at 3,500 tons/year and 22,000 tons/years capacity', Intern. Symp Env. Ind, Bucharest, 18-20.

Zhang, C, Khoshmanesh, K, Mitchell, A \& Kalantar-zadeh, K 2010, 'Dielectrophoresis for manipulation of micro/nano particles in microfluidic systems'. Analytical and bioanalytical chemistry, vol. 396, no. 1, pp. 401-420.

Zhiqiang, Q, Siegmann, K, Keller, A, Matter, U, Scherrer, L \& Siegmann, H 2000 , 'Nanoparticle air pollution in major cities and its origin'. Atmospheric Environment, vol. 34, no. 3, pp. 443-451. 